\title{
Central antinociceptive activity of peripherally applied botulinum toxin type $A$ in lab rat model of trigeminal neuralgia
}

\author{
Chuanjie Wu ${ }^{\dagger}$, Nanchang Xie ${ }^{\dagger}$, Yajun Lian ${ }^{*}$, Hongliang Xu, Chen Chen, Yake Zheng, Yuan Chen \\ and Haifeng Zhang
}

\begin{abstract}
Background: BoNT-A is often used in the clinical treatment for movement disorders. In recent years, various clinical studies suggest that BoNT-A can effectively alleviate pain caused by trigeminal neuralgia (TN); however, its mechanism remains unclear.

Methods: In this study, we used a lab rat model for TN produced by chronic constriction injury of the infraorbital nerve (ION-CCI). Restrained rats were injected subcutaneously with BoNT-A into the whisker pad tissue (ipsilaterally to the nerve injury) 14 days after the ION-CCl. Allodynia was tested by Von Frey filaments and TRPs and CSNAP-25 were tested by western blot.

Results: Peripheral application of BoNT-A (3,10 U/kg) significantly increased the pain threshold of ION-CCI rats. Rota-rod test showed that BoNT-A administration at doses tested did not significantly affect rat motor coordination. By probing for a specific marker for BoNT-A, cleaved synaptosomal-associated protein 25 (cSNAP-25), we found that peripheral application of BoNT-A (10 U/kg) affected brainstem Vc, which could be blocked by the axonal transport blocker colchicine. In addition, western blot analysis showed that in the $\mathrm{Vc}$ region of ION-CCI rats, the expression levels of TRPA1, TRPV1, TRPV2 and TRPM8 increased, whereas peripheral application of BoNT-A significantly lowered the high expression of TRPA1, TRPV1 and TRPV2, but not TRPM8 at 7 days after BoNT-A injection.

Conclusions: The finding of this study suggest that peripherally applied BoNT-A can produce antinociceptive effects in ION-CCI model. The underlying mechanisms may be BoNT-A acts on the Vc via axonal transport, inhibits the high expression of TRPA1, TRPV1 and TRPV2, and reduces central sensitization.
\end{abstract}

Keywords: Trigeminal neuralgia, Botulinum toxin type A, Central antinociceptive activity, Rat

\section{Background}

Trigeminal neuralgia (TN) is episodic facial pain that is usually described to feel like a unilateral electric shock. This neuropathic disorder has been shown to be profoundly distressing and to negatively impact the patient's well-being (Hall et al. 2006). According to epidemiological studies, approximately 4-28.9/100,000 persons worldwide experience TN (Hall et al. 2006; Dieleman

\footnotetext{
*Correspondence: lianyajun369@yahoo.com

${ }^{\dagger}$ Chuanjie Wu and Nanchang Xie contributed equally to this work Department of Neurology, the First Affiliated Hospital of Zhengzhou University, Zhengzhou 450052, China
}

et al. 2008; Katusic et al. 1990). Patients with TN usually present a clinical treatment challenge. The antiepileptic drugs are usually used first in an attempt to treat TN. However, treatment with antiepileptic drugs results in more adverse reactions, and requires daily administration. In addition, long-term use can cause a gradual decline of drug efficacy (Taylor et al. 1981).

Botulinum toxin type A (BoNT-A) is one of the serotypes (A, B, C1, C2, D, E, F and G) of botulinum neurotoxins derived from Clostridium botulinum (Setler 2002). Brin et al. (1987) reported the use of BoNT-A for treatment of dystonia, which results in relief of dystonia symptoms, as well as significant pain experience

\section{Springer Open}


improvement in $74 \%$ of the patients. Subsequently, the antinociceptive effects of BoNT-A are gradually recognized (Luvisetto et al. 2015). With in-depth understanding, several clinical studies indicate that BoNT-A can effectively alleviate TN (Zuniga et al. 2008; Ngeow and Nair 2010; Bohluli et al. 2011). In 2012, we first used the RCT experimental method to demonstrate that BoNT-A can effectively alleviate the pain caused by $\mathrm{TN}$ with mild adverse reactions (Wu et al. 2012). Subsequent studies further confirm the effectiveness of BoNT-A for the treatment of TN (Zhang et al. 2014; Xia et al. 2016; Li et al. 2014). However, the mechanism of BoNT-A treatment for TN remains unclear. Currently, most studies on the mechanism of the antinociceptive effects of botulinum toxin focus on the formalin-induced pain model, as well as pre-application of BoNT-A to explore its role in pain prevention (Cui et al. 2004).

As most case of TN are caused by sensory nerve root compression (Zakrzewska and Linskey 2014), Vos et al. (1994) developed a lab rat model of TN produced by chronic constriction injury of the infraorbital nerve (ION-CCI), which is a branch of the trigeminal nerve. This model reproduces important aspects of TN, including signs of abnormal spontaneous pain-related behavior and mechanical allodynia (Vos et al. 1994).

The aim of the present study is to investigate the antinociceptive effects of BoNT-A in the rat ION-CCI model, and whether BoNT-A exerts antinociceptive function by acting on the central nervous system. In addition, we also examined the potential central antinociceptive mechanisms of BoNT-A.

\section{Methods}

\section{Animals and trigeminal neuralgia model}

Adult male Sprague-Dawley rats (Experimental Animal Center of Zhengzhou University) weighing 220-300 were used. All rats were housed in climate-controlled rooms on a 12/12 light/dark cycle with water and standardized rodent diet available ad libitum. The experimental procedures were approved by the Commission of Zhengzhou University for ethics of experiments on animals in accordance with international standards (No. YFY2015096).

The trigeminal neuralgia model was performed by the method as described previously (Imamura et al. 1997). Animals were anesthetized by a single intraperitoneal injection of $10 \%$ chloral hydrate $(0.2 \mathrm{ml} / \mathrm{kg})$. All incisions were made intraorally, which allowed the hair on the snout and vibrissae to remain intact. An incision approximately $10 \mathrm{~mm}$ long was made along the gingivobuccal margin. The incision was placed proximal to the first molar. The infraorbital nerve was dissected and two chromic catgut ligatures (4-0) were placed around the nerve spaced $2 \mathrm{~mm}$ apart. The ligatures reduced the diameter of the nerve by just a noticeable amount and they did not interrupt the epineural circulation. The incision was sutured at three points using 4.0 silk. The sham operation was identical except that the ION was not ligated.

\section{Drug administration}

BoNT-A (Hengli, Lanzhou, China) was reconstituted in adequate volume of $0.9 \%$ saline. Restrained rats were injected subcutaneously with BoNT-A $(30 \mu \mathrm{l})$ into the whisker pad tissue (ipsilaterally to the nerve injury) 14 days after the ION-CCI using a Hamilton syringe needle (Hamilton Microliter 801, Hamilton, Bonaduz, Switzerland). The dosed used were 3 , and $10 \mathrm{U} / \mathrm{kg}$ BoNT-A, respectively. For control rats, $30 \mu \mathrm{l}$ normal saline was injected.

Colchicine (Saxama, Wuhan, China) was reconstituted in normal saline to obtain the $5 \mathrm{mM}$ concentration. Colchicine or normal saline $(2 \mu \mathrm{l})$ was injected into the trigeminal ganglion (ipsilaterally to the nerve injury) of anesthetized rat as described previously (Neubert et al. 2005). The Hamilton syringe needle (Hamilton Microliter 801, Hamilton, Bonaduz, Switzerland) was briefly inserted medially $(1-2 \mathrm{~mm})$ to the palpated portion of the zygomatic process through the infraorbital foramen. The needle was positioned at a 10 degree angle relative to the midline of the head. The tip of the needle was advanced approximately $20 \mathrm{~mm}$ along the infraorbital canal and subsequently through the foramen rotundum then the colchicines or isometric normal saline was injected.

\section{Mechanical allodynia testing}

Von Frey hairs (North coast medical Inc, Morgan Hill, CA) were used for mechanical stimulation. The filaments produced a bending force of $0.008,0.02,0.04,0.07,0.16$, $0.4,0.6,1.0,1.4,2.0,4.0,6.0,8.0,10.0,15.0,26.0,60.0$, 100, 200 and $300 \mathrm{~g}$. Testing was performed as previously described in detail by Vos et al. (1994). In brief, a single rat is placed in a small transparent plastic cage for $10 \mathrm{~min}$ to accommodate to the experimental environment until they assumed their normal sniffing/no locomotion position. Testers were not informed as to which rats had CCI or sham surgery. After the acclimatization sessions the tester was able to apply the von Frey hair to the ipsilateral territory of the injury ION (whisker pad), starting at $0.008 \mathrm{~g}$, until a defined behavioral response was elicited. When rats showed positive reactions, the intensity was recorded, which was the pain threshold. Positive reactions included (1) withdrawal reaction with rapid head 
withdraw; (2) escape or aggressive behavior, manifested as escape, curling up, hiding its head, or biting or grasping the stimulant; (3) asymmetric face grooming, rat displays an uninterrupted series of at least three face-wash strokes directed to the stimulated facial area. If rats did not show any of the aforementioned reactions at the stimulation intensity of $26 \mathrm{~g}$, then the pain threshold was given $26 \mathrm{~g}$. Measurements were performed three times in ten min intervals and the mean of the measurements is used.

\section{Rota-rod test}

To investigate whether BoNT-A has a systemic effect, we tested the effect of BoNT-A (at dose of 3 and $10 \mathrm{U} / \mathrm{kg}$ ) on rat's performance on the Rota-rod test. For the Rota-rod experimental apparatus (Ugo Basile, Italy), the rod diameter was $6 \mathrm{~cm}$, and the rotating speed was set to gradually increase from 5 to $40 \mathrm{r} / \mathrm{min}$ in $5 \mathrm{~min}$. The latency for the rat to fall from the rotating rod was recorded. Each rat was subjected to four consecutive tests and results were averaged.

\section{Western blot}

The caudal subnucleus of the spinal trigeminal nucleus (Vc) was collected from deeply anesthetized, rapidly decapitated rats at designated time, either proceeded immediately for biochemical studies or kept at $-80{ }^{\circ} \mathrm{C}$ until use. Western blot was performed as described previously (Xie et al. 2014). Briefly, $30 \mu \mathrm{g}$ of protein was separate by SDS-polyacrylamide gel electrophoresis (PAGE) and then transferred to PVDF membranes. After blocking in $5 \%$ fat-free milk in Tris-buffered saline containing $0.1 \%$ Tween, immunoblots were probed with antibodies to cleaved synaptosomal-associated protein 25 (cSNAP-25) (1:2000; GeneTex, USA), transient receptor potential ankyrin 1 (TRPA1) (1:3000; Abcame, UK), transient receptor potential vanilloid type 1 (TRPV1) (1:1000, Sigma, USA), transient receptor potential vanilloid type 2 (TRPV2) (1:1500; Sigma, USA) and transient receptor potential vanilloid melastatin 8 (TRPM8) (1:1000; Abcame, UK). The same blots were stripped and reprobed with antibodies to $\beta$-actin (1:5000; Santa Crus, USA). The blots were then incubated with horseradish peroxidase (HRP)-conjugated secondary antibody (1:10,000, Boster, Wuhan, China) for $1 \mathrm{~h}$ at $37^{\circ} \mathrm{C}$. Immunoreactivity was visualized by chemiluminescence and exposure to a film. Band intensities were quantified by densitometric analysis using a densitometer.

\section{Statistical analysis}

All data is expressed as mean $\pm \mathrm{SD}$. The statistical significance was assessed using One-way ANOVA and the New-man-Keuls test. All statistical analyses were performed using SPSS 18.0. $\mathrm{P}<0.05$ was considered statistically significant.

\section{Results}

Antinociceptive effects of peripherally applied BoNT-A

Von Frey hairs were used to examine the antimociceptive effects of BoNT-A. Our results showed that 14 days after ION-CCI, the ipsilateral pain threshold of the IONCCI group was significantly reduced compared to the sham-operated group $(\mathrm{P}<0.05)$. BoNT-A $(3,10 \mathrm{U} / \mathrm{kg})$ as injected subcutaneously into the ipsilateral whisker pad tissue 14 days after the ION-CCI. Four days after the subcutaneous injection with BoNT-A, pain thresholds of each BoNT-A dose group significantly increased compared to the control group $(\mathrm{P}<0.05)$. This antimociceptive effects reached a maximum level at 8 days and remained significantly elevated until 20 days. The $10 \mathrm{U} /$ $\mathrm{kg}$ group had better antimociceptive effects than the $3 \mathrm{U} /$ $\mathrm{kg}$ group; however, the difference between them was not statistically significant $(\mathrm{P}>0.05)$ (Fig. 1).

\section{Effects of BoNT-A on rat motor coordination ability}

BoNT-A (3, $10 \mathrm{U} / \mathrm{kg})$ injected subcutaneously into the ipsilateral whisker pad did not affect the motor coordination ability of rats. Rota-rod test is a commonly used method to reflect the motor coordination ability of rats. Results of the Rota-rod test showed that 7 days after injection, the averaged latencies of rats to fall from the rotating rod of the two dose groups were not statistically significantly different from those of the control group (Table 1).

\section{SNAP-25 cleavage in Vc and central antinociceptive effects after BoNT-A peripheral application is axonal transport dependent}

Among the other Botulinum toxins, BoNT-A cleaves specific sites of synaptosomal-associated protein 25 (SNAP25 ) to inhibit the exocytosis of neurotransmitters from the nerve terminals. So, the cSNAP-25 can be used as a reliable marker of the BoNT-A diffusion and local action. Fourteen days after ION-CCI operation, BoNT-A (10 U/ $\mathrm{kg}$ ) injection was performed. Seven days after BoNT-A injection, the level of cSNAP-25 in the Vc in the BoNT-A treatment group significantly increased compared to control group $(\mathrm{P}<0.05)$. When axonal transport blocker colchicine was injected ipsilateral to BoNT-A injection into the trigeminal ganglion $12 \mathrm{~h}$ before the BoNT-A treatment, BoNT-A $(10 \mathrm{U} / \mathrm{kg})$ failed to increase the level of cSNAP-25 (P > 0.05) in the Vc (Fig. 2). Behavioral studies also indicated that compared to the control group, ipsilateral colchicine injection into the trigeminal ganglion resulted in disappearance of the antinociceptive effects of BoNT-A (10 U/kg) (P > 0.05) (Fig. 3). 


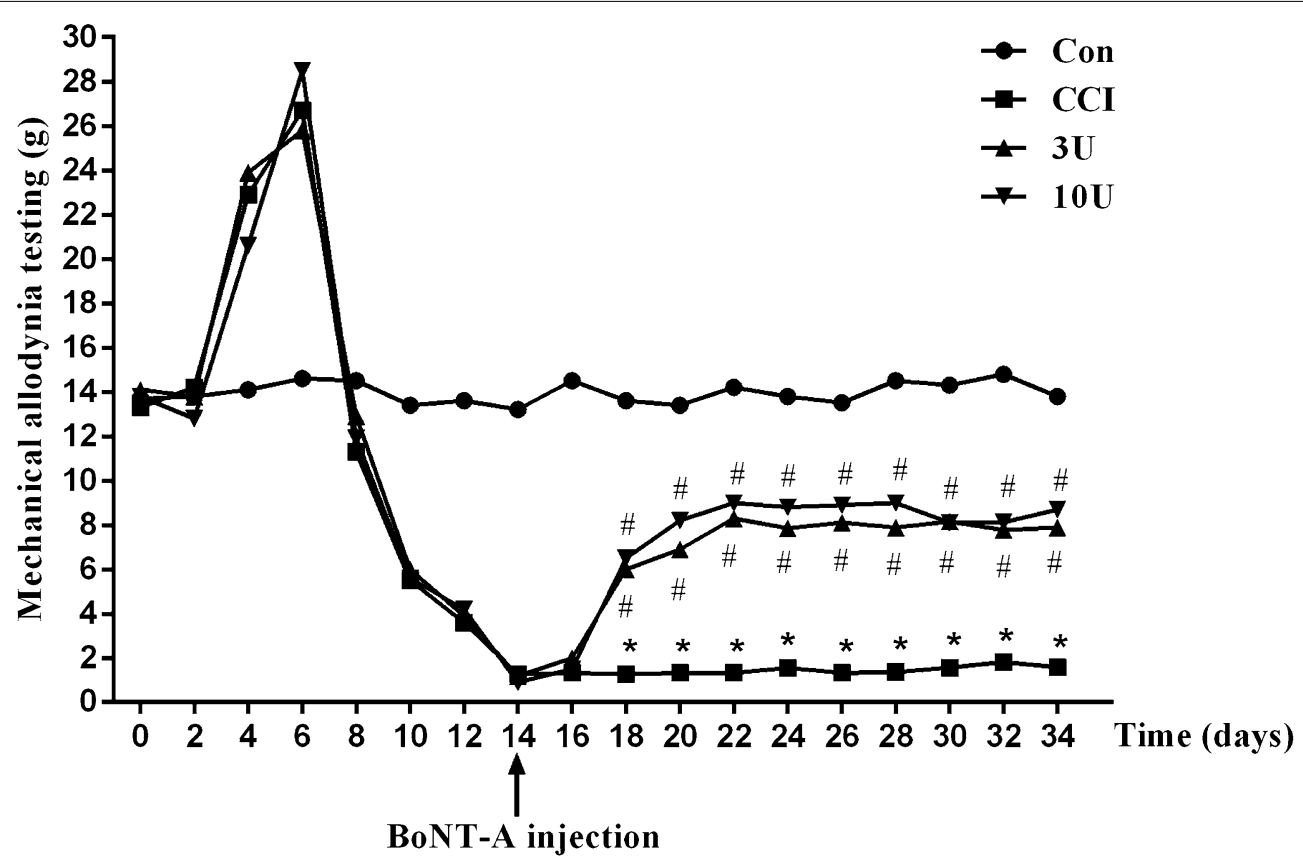

Fig. 1 Effect of BoNT-A on mechanical hypersensitivity induced by ION-CCI. Rats underwent either ION-CCl or sham operation. Fourteen days after the operation, BoNT-A $(3,10 \mathrm{U} / \mathrm{kg})$ or normal saline was injected into the whisker pad ipsilateral to the operation site. Measurement were performed on the ipsilateral to the nerve injury. ${ }^{*} \mathrm{P}<0.05$ versus control group and ${ }^{\#} \mathrm{P}<0.05$ versus $\mathrm{CCl}$ group

Table 1 Effects of BoBT-A on rat motor coordination ability (accessed by Rota-rod test)

\begin{tabular}{lll}
\hline & $\mathbf{3} \mathbf{U}(\mathbf{s})$ & $\mathbf{1 0 U}(\mathbf{s})$ \\
\hline Before BoNT-A treatment & $268.2 \pm 13.8$ & $271.8 \pm 16.6$ \\
Seven days after BoNT-A treatment & $255.7 \pm 8.5^{*}$ & $278.6 \pm 11.4^{*}$ \\
\hline
\end{tabular}

* $P>0.05$ versus Before BoNT-A treatment

\section{Effect of BoNT-A on TRPs expression in Vc tissues}

To obtain insight into the mechanisms of antinociceptive effects of BoNT-A, the protein expression of TRPA1, TRPV1, TRPV2 and TRPM8 was further examined in ION-CCI model of trigeminal neuralgia. Western blot demonstrated that TRPA1 and TRPV1 expression increased remarkable at 14 days $(\mathrm{P}<0.05)$ after IONCCI and increased until 28 days $(\mathrm{P}<0.05)$, TRPV2 increased remarkable at 7 days $(\mathrm{P}<0.05)$ after ION-CCI and increased until 28 days $(\mathrm{P}<0.05)$, TRPM8 expression started to increase at 7 days $(\mathrm{P}<0.05)$ after ION-CCI, reached a maximum level at 14 and remained significantly elevated until 28 days $(\mathrm{P}<0.05)$ (Fig. 4a). While subcutaneously administration of BoNT-A (3, $10 \mathrm{U} /$ $\mathrm{kg}$ ) significantly decreased the expression of TRPA1 and TRPV1 at 7 days after BoNT-A injection in a doserelated manner $(\mathrm{P}<0.05)$. BoNT-A $(10 \mathrm{U} / \mathrm{kg}$ rather than $3 \mathrm{U} / \mathrm{kg}$ ) significantly decreased the expression of TRPV2

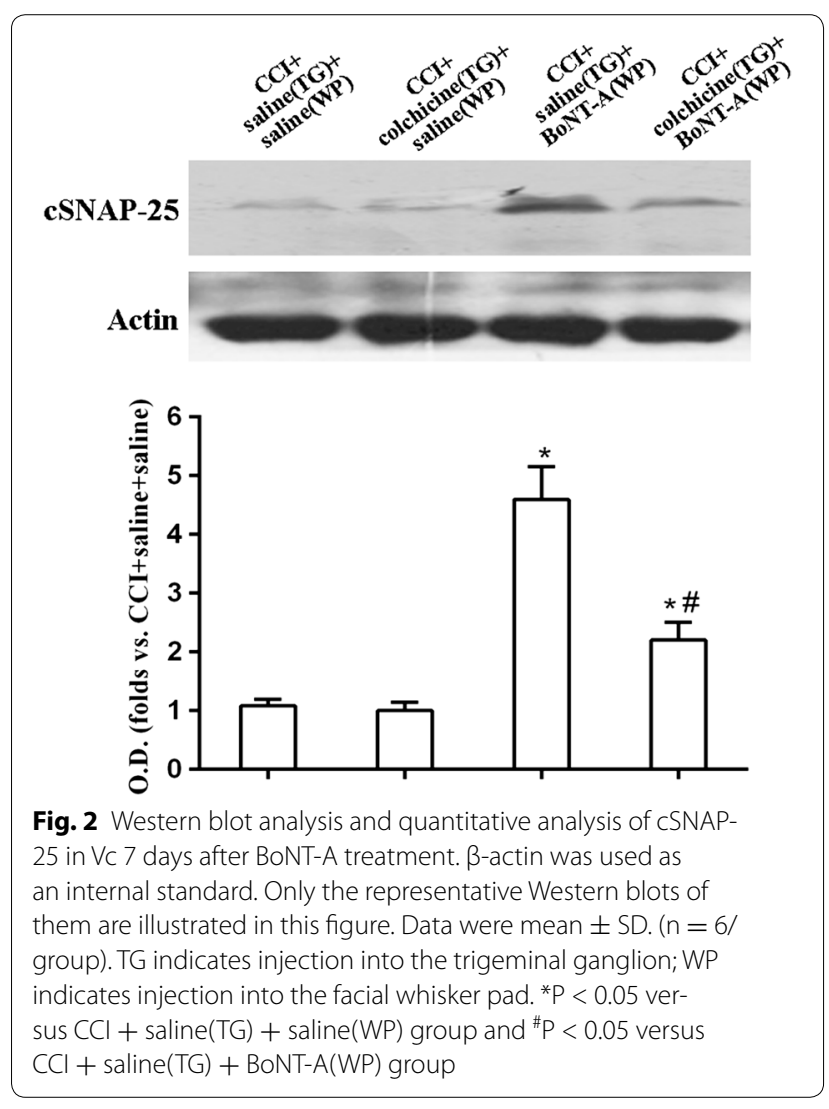




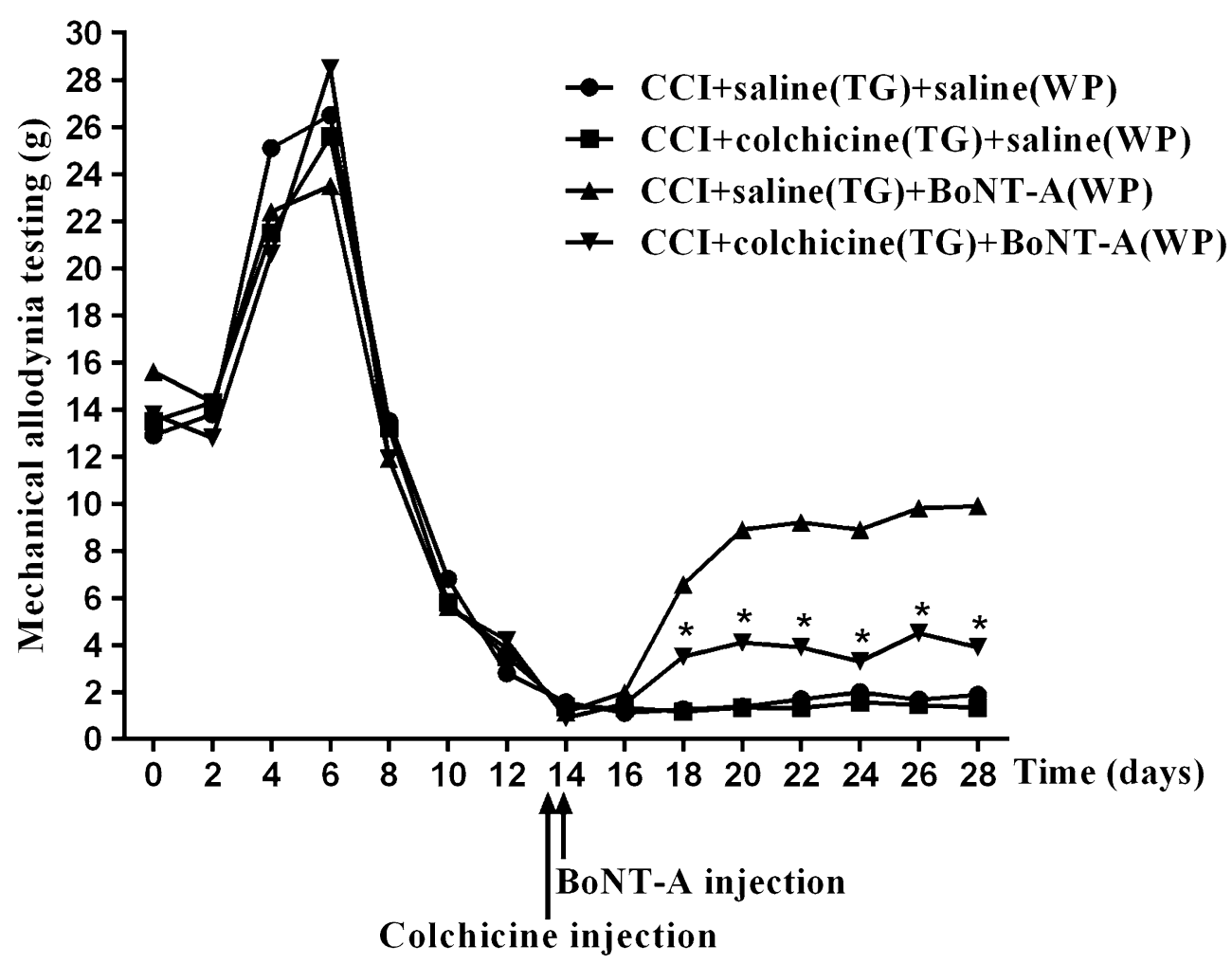

Fig. 3 Axonal transport blocker colchicine blocked antinociceptive effects of BoNT-A (10 U/kg). Rats underwent either ION-CCl or sham operation. Injection of BoNT-A $(3,10 \mathrm{U} / \mathrm{kg})$ or normal saline into the whisker pad ipsilateral to the operation site was performed 14 days after the operation. Colchicine or normal saline was injected into ipsilateral trigeminal ganglion $12 \mathrm{~h}$ before the application of BoNT-A. TG indicates injection into the trigeminal ganglion; WP indicates injection into the facial whisker pad. *P $<0.05$ versus $\mathrm{CCl}+$ saline(TG) + BoNT-A(WP) group

at 7 days after BoNT-A injection. TRPM8 expression was not change significantly after BoNT-A $(3,10 \mathrm{U} / \mathrm{kg})$ treatment compared with control group (P > 0.05) (Fig. 4b).

\section{Discussion}

The application of BoNT-A has been recently explored in a number of pain associated disorders, such as diabetic neuropathy, complex regional pain syndrome, trigeminal neuralgia, and occipital neuralgia (Oh and Chung 2015). In recent years, a number of clinical studies have shown that BoNT-A treatment for $\mathrm{TN}$ is safe and effective (Zuniga et al. 2008; Ngeow and Nair 2010; Wu et al. 2012; Zhang et al. 2014; Li et al. 2014). However, the intrinsic limitations of clinical studies hamper the in-depth analysis on its mechanism. In recent years, researchers have explored the treatment and mechanism of BoNT-A for pain associated with trigeminal nerve region (Matak et al. 2011; Kim et al. 2015). However, these studies essentially use the formalin-induced inflammatory pain model and BoNT-A pretreatment method to study the mechanism. The features of formalin-induced inflammatory pain model are inconsistent with those of $\mathrm{TN}$. In addition,
BoNT-A pretreatment method is not a good clinical simulation of BoNT-A treatment for TN. The ION-CCI model is widely accepted as an appropriate model of trigeminal neuralgia (Vos et al. 1994). In this study, we used the ION-CCI model of TN and examined the antinociceptive effects of BoNT-A in successfully generated model, which is a good animal model for studying the clinical BoNT-A treatment for TN.

In this study, we found that BoNT-A significantly increased the mechanical stimulation threshold in rat ION-CCI model of trigeminal neuralgia, which is similar to the results observed in a previous study (Filipovic et al. 2012). However, most previous studies on the IONCCI model of TN use BoNT-A doses based on the doses used in other pain models. In this study, we found that differences in antinociceptive effects between different doses of BoNT-A in ION-CCI model of TN were not statistically significant, which is similar to the results of our previous clinical studies that there is no statistically significant differences in clinical efficacy between lowdose $(25 \mathrm{U})$ and high-dose (75U) of BoNT-A treatment in TN patients (Zhang et al. 2014). This also suggests that 

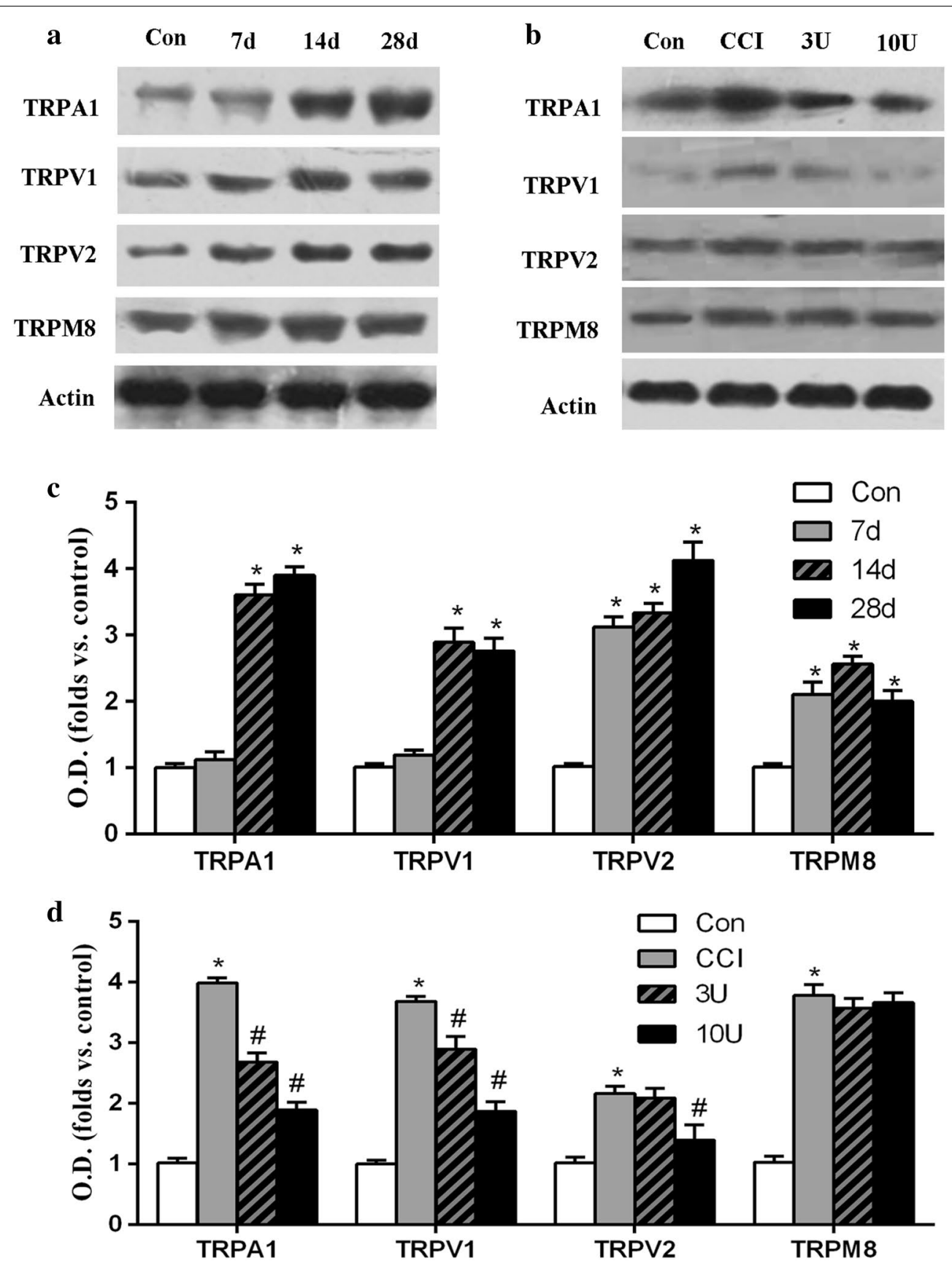

Fig. 4 The protein levels of TRPS. a, c Western blots analysis and quantitative of TRPA1, TRPV1, TRPV2 and TRPM8 at various times after ION-CCI. b, $\mathbf{d}$ Western blots analysis and quantitative of TRPA1, TRPV1, TRPV2 and TRPM8 at 7 days after BoNT-A or normal saline injection (21 days after operation) in 4 treatment groups. $\beta$-actin was used as an internal standard. Only the representative Western blots of them are illustrated in this figure. Data were mean $\pm S D$ ( $n=6 /$ group). TG indicates injection into the trigeminal ganglion; WP indicates injection into the facial whisker pad. ${ }^{*} P<0.05$ versus control and ${ }^{\#} \mathrm{P}<0.05$ versus $\mathrm{CCl}$ group

the animal model and experimental method used in this study are consistent with the features of clinical BoNT-A treatment for TN.

The treatment mechanism of BoNT-A for TN is currently unclear. Most previous studies suggest that
BoNT-A acts locally or on the trigeminal ganglia (Cui et al. 2004; Xiao et al. 2013). Vc is the primary relay for orofacial pain and temperature sensations and the site for processing sensory information, and plays an important role in the mechanism of $\mathrm{TN}$ pathogenesis. In this study, 
we used a specific BoNT-A marker, cSNAP-25, to determine the possible sites of BoNT-A action in the ION-CCI model of TN. By combining colchicine injection to block axonal transport, we proved that BoNT-A exerts antinociceptive effects in brainstem Vc via axonal transport in the ION-CCI model of TN. The results are similar to the BoNT-A study results in other models (Matak et al. 2011, 2012). Matak et al. inject BoNT-A locally in the sciatic nerve area and detect cSNAP-25 in the corresponding spinal cord sections.

Since BoNT-A acts on the central nervous system through axonal transport, we examined whether BoNTA affects the motor coordination ability in rats. To the best of our knowledge, this study first used Rota-rod test to demonstrate that BoNT-A injection into facial trigeminal nerve region did not cause systemic effects in rats even at high doses $(10 \mathrm{U} / \mathrm{kg})$. This suggests that BoNTA exerts specific antinociceptive function in the central nervous system without affecting its other functions.

In recent years, TRPs have been identified as nonselective cation channel proteins localized in the plasma membrane and membranes of intracellular organelles. A significant difference between TRPs family proteins and other ion channel family proteins is that members of TRP family share low homology and can be activated or sensitized by a variety of mediators and ligands. It is currently recognized that TRPA1, TRPV1, TRPV2 and TRPM8 play an important role in the pathogenesis of pain sensation production and hyperalgesia (Ferrandiz-Huertas et al. 2014), and are involved in the perception of pain induced by chemical, temperature or mechanical stimuli (Mickle et al. 2015). Most previous studies on ION-CCI model of TN focus on pathological changes of trigeminal ganglia. To the best of our knowledge, this study first demonstrated that TRPA1, TRPV1, TRPV2 and TRPM8 expression elevated in the $\mathrm{Vc}$ in ION-CCI model of TN, and BoNT-A effectively inhibited the high expression of TRPA1, TRPV1 and TRPV2. This suggests that BoNT-A is able to reduce central sensitization and therefore exerts antinociceptive function by inhibiting the high expression of nociceptors, such as TRPA1, TRPV1 and TRPV2. In addition, we also found that BoNT-A had no effect on the increased expression of TRPM8, hence suggesting BoNT-A does not affect TRPM8 expression. However, the effects of BoNT-A on TRPM8 require further study to confirm.

\section{Conclusions}

In conclusion, our findings suggest that peripherally applied BoNT-A can produce antinociceptive effects in ION-CCI model. The underlying mechanisms may be BoNT-A directly acts on the Vc via axonal transport, inhibits the high expression of TRPA1, TRPV1 and
TRPV2, and reduces central sensitization. This study provides not only a theoretical basis for clinical application of BoNT-A for TN treatment and other pain associated disorders, but also a new direction for understanding the antinociceptive mechanism of BoNT-A.

\section{Authors' contributions}

CW, NX and YL participated in the design of the study, analysis of the data and wrote the draft of the manuscript. HX participated in the design of the study and have made substantial contributions to the acquisition of data and also revised the manuscript critically for important intellectual content. CC and YZ participated in the design of the study, analyzed the data and performed the statistical analysis besides helping the revisions. YC and $\mathrm{HZ}$ participated in the design of the study and have been involved in revising the manuscript critically for important intellectual content. All authors read and approved the final manuscript.

\section{Acknowledgements}

This work was supported by a grant from National Natural Science Foundation of China (Nos. U1404809, 81571260) and the Youth Innovation Fund of the First Affiliated Hospital of the Zhengzhou University.

\section{Competing interests}

The authors declare that they have no competing interests.

Received: 6 February 2016 Accepted: 27 March 2016

Published online: 11 April 2016

\section{References}

Bohluli B, Motamedi MH, Bagheri SC, Bayat M, Lassemi E, Navi F, Moharamnejad N (2011) Use of botulinum toxin a for drug-refractory trigeminal neuralgia: preliminary report. Oral Surg Oral Med Oral Pathol Oral Radiol Endod 111:47-50

Brin MF, Fahn S, Moskowitz C, Friedman A, Shale HM, Greene PE, Blitzer A, List T, Lange D, Lovelace RE et al (1987) Localized injections of botulinum toxin for the treatment of focal dystonia and hemifacial spasm. Mov Disord 2:237-254

Cui M, Khanijou S, Rubino J, Aoki KR (2004) Subcutaneous administration of botulinum toxin a reduces formalin-induced pain. Pain 107:125-133

Dieleman JP, Kerklaan J, Huygen FJ, Bouma PA, Sturkenboom MC (2008) Incidence rates and treatment of neuropathic pain conditions in the general population. Pain 137:681-688

Ferrandiz-Huertas C, Mathivanan S, Wolf CJ, Devesa I, Ferrer-Montiel A (2014) Trafficking of thermotrp channels. Membranes 4:525-564

Filipovic B, Matak I, Bach-Rojecky L, Lackovic Z (2012) Central action of peripherally applied botulinum toxin type a on pain and dural protein extravasation in rat model of trigeminal neuropathy. PLoS One 7:e29803

Hall GC, Carroll D, Parry D, McQuay HJ (2006) Epidemiology and treatment of neuropathic pain: the UK primary care perspective. Pain 122:156-162

Imamura Y, Kawamoto H, Nakanishi O (1997) Characterization of heat-hyperalgesia in an experimental trigeminal neuropathy in rats. Exp Brain Res 116:97-103

Katusic S, Beard CM, Bergstralh E, Kurland LT (1990) Incidence and clinical features of trigeminal neuralgia, rochester, minnesota, 1945-1984. Ann Neurol 27:89-95

Kim HJ, Lee GW, Kim MJ, Yang KY, Kim ST, Bae YC, Ahn DK (2015) Antinociceptive effects of transcytosed botulinum neurotoxin type a on trigeminal nociception in rats. Korean J Physiol Pharmacol 19:349-355

Li S, Lian YJ, Chen Y, Zhang HF, Ma YQ, He CH, Wu CJ, Xie NC, Zheng YK, Zhang $Y$ (2014) Therapeutic effect of botulinum toxin-a in 88 patients with trigeminal neuralgia with 14-month follow-up. J Headache Pain 15:43

Luvisetto S, Gazerani P, Cianchetti C, Pavone F (2015) Botulinum toxin type a as a therapeutic agent against headache and related disorders. Toxins 7:3818-3844

Matak I, Bach-Rojecky L, Filipovic B, Lackovic Z (2011) Behavioral and immunohistochemical evidence for central antinociceptive activity of botulinum toxin a. Neuroscience 186:201-207 
Matak I, Riederer P, Lackovic Z (2012) Botulinum toxin's axonal transport from periphery to the spinal cord. Neurochem Int 61:236-239

Mickle AD, Shepherd AJ, Mohapatra DP (2015) Sensory trp channels: the key transducers of nociception and pain. Prog Mol Biol Transl Sci 131:73-118

Neubert JK, Mannes AJ, Keller J, Wexel M, ladarola MJ, Caudle RM (2005) Peripheral targeting of the trigeminal ganglion via the infraorbital foramen as a therapeutic strategy. Brain Res Protoc 15:119-126

Ngeow WC, Nair R (2010) Injection of botulinum toxin type a (botox) into trigger zone of trigeminal neuralgia as a means to control pain. Oral Surg Oral Med Oral Pathol Oral Radiol Endod 109:e47-e50

Oh HM, Chung ME (2015) Botulinum toxin for neuropathic pain: a review of the literature. Toxins 7:3127-3154

Setler PE (2002) Therapeutic use of botulinum toxins: background and history. Clin J Pain 18:S119-S124

Taylor JC, Brauer S, Espir ML (1981) Long-term treatment of trigeminal neuralgia with carbamazepine. Postgrad Med J 57:16-18

Vos BP, Strassman AM, Maciewicz RJ (1994) Behavioral evidence of trigeminal neuropathic pain following chronic constriction injury to the rat's infraorbital nerve. J Neurosci 14:2708-2723
Wu CJ, Lian YJ, Zheng YK, Zhang HF, Chen Y, Xie NC, Wang LJ (2012) Botulinum toxin type a for the treatment of trigeminal neuralgia: results from a randomized, double-blind, placebo-controlled trial. Cephalalgia 32:443-450

Xia JH, He CH, Zhang HF, Lian YJ, Chen Y, Wu CJ, Ma YQ (2016) Botulinum toxin a in the treatment of trigeminal neuralgia. Int J Neurosci 126:348-353

Xiao L, Cheng J, Zhuang Y, Qu W, Muir J, Liang H, Zhang D (2013) Botulinum toxin type A reduces hyperalgesia and TRPV1 expression in rats with neuropathic pain. Pain Med 14:276-286

Xie N, Wang C, Lian Y, Wu C, Zhang H, Zhang Q (2014) Puerarin protects hippocampal neurons against cell death in pilocarpine-induced seizures through antioxidant and anti-apoptotic mechanisms. Cell Mol Neurobiol 34:1175-1182

Zakrzewska JM, Linskey ME (2014) Trigeminal neuralgia. BMJ 348:9474

Zhang H, Lian Y, Ma Y, Chen Y, He C, Xie N, Wu C (2014) Two doses of botulinum toxin type a for the treatment of trigeminal neuralgia: observation of therapeutic effect from a randomized, double-blind, placebo-controlled trial. J Headache Pain 15:65

Zuniga C, Diaz S, Piedimonte F, Micheli F (2008) Beneficial effects of botulinum toxin type a in trigeminal neuralgia. Arq Neuropsiquiatr 66:500-503

\section{Submit your manuscript to a SpringerOpen ${ }^{\circ}$ journal and benefit from:}

- Convenient online submission

- Rigorous peer review

- Immediate publication on acceptance

- Open access: articles freely available online

- High visibility within the field

- Retaining the copyright to your article

Submit your next manuscript at $>$ springeropen.com 